\title{
1 Title: The content and structure of reputation domains across \\ 2 human societies: a view from the evolutionary social sciences
}

3 Authors: Zachary H. Garfield ${ }^{13}$, Ryan Schacht ${ }^{2}$, Emily R. Post ${ }^{3}$, Dominique Ingram ${ }^{3}$, Andrea

4 Uehling ${ }^{3}$, and Shane J. Macfarlan ${ }^{3 \mathrm{a}}$

5

6

\author{
${ }^{1}$ Institute for Advanced Study in Toulouse, Université de Toulouse 1 Capitole, Toulouse, \\ France; ${ }^{2}$ Department of Anthropology, East Carolina University, Greenville, NC, USA; ${ }^{3}$ \\ Department of Anthropology, University of Utah, Salt Lake City, UT, USA \\ ${ }^{a}$ Corresponding authors
}

Keywords: Reputation, Prosociality, Cooperation, Human Uniqueness, Cross-Cultural Analysis

Preprint manuscript also archived at Zendo (https://doi.org/10.5281/zenodo.4742375).

4

\section{Abstract}

Reputations are an essential feature of human sociality and the evolution of cooperation and group living. Much scholarship has focused on reputations, yet typically on a narrow range of domains (e.g., prosociality, aggressiveness), usually in isolation. Humans can develop reputations, however, from any collective information. We conducted exploratory analyses on the content, distribution, and structure of reputation domain diversity across cultures, using the Human Relations Area Files ethnographic database. After coding ethnographic texts on reputations from 153 cultures, we used hierarchical modelling, cluster analysis, and text analysis to provide an empirical view of reputation domains across societies. Findings suggest: 1) reputational domains vary cross-culturally, yet reputations for cultural conformity, prosociality, social status, and neural capital are widespread; 2) reputation domains are more variable for males than females; and 3) particular reputation domains are interrelated, demonstrating a structure consistent with dimensions of human uniqueness. We label these features: Cultural group unity, Dominance, Neural capital, Sexuality, Social and material success, and Supernatural healing. We highlight the need for future research on the evolution of cooperation and human sociality to consider a wider range of reputation domains, as well as their social, ecological, and gender-specific variability. 
34 Reputations are essential for human sociality. Whether used to punish norm violators in small communities or orient behaviour in anonymous online markets, reputations matter [1]. Reputations represent collective beliefs and evaluations a community forms about an individual's behavioural or emotional tendencies [2,3]. They function as currencies in a social marketplace with individuals signalling qualities relative to peers $[4,5]$. Such signals can reduce transaction costs in the formation, maintenance, and termination of relationships by providing information about others without direct experience [6]. Because reputations can facilitate prosocial behaviour and punish deviancy, they provide some cognitive scaffolding supporting human sociality, including, the formation of status hierarchies [7,8], social institutions [9], and prosociality [10,11]. Many species rely on reputation-information exchange [12]. Among humans, however, language and gossip creates a selective environment whereby reputations have significant social consequences [13-15].

Individual reputations can develop for any domain in which collective information exists on people's behavioural or emotional tendencies [16]. As new formats of social interaction emerge, the human behavioural repertoire becomes unbounded [17], suggesting an unlimited number of potential reputation domains. Nevertheless, evolutionary scholars have typically focused on a narrow range of reputation domains, such as prosociality [14,18-21], competency [3,22,23], aggressiveness [24-26], and sexuality [27,28]. This research has produced valuable insights on the influence of particular reputation domains on facets of social interaction [4], gendered relationships [28], and the evolution of social systems [29].

Research on reputations has remained agnostic, however, about the scope of reputation domains within societies, their frequency across cultures, and potential gender biases [30]. Furthermore, research has often occurred in a piece-meal fashion focusing on a single domain, obfuscating the degree to which domains interact and shape behavioural responses as a suite of integrated parts (however, see [3]). Current scholarship lacks a clear understanding of the content, structure, and diversity of reputation domains across

62 societies.

63 We seek to build a foundation for comparative approaches to reputation domain diversity through exploratory analyses of the ethnographic record. We first derive a list of a priori reputation domains (discussed in the Supplementary Information [SI]). We then assess the cross-cultural frequency of evidence for reputation domains and how evidence for genderspecific reputations varies. Lastly, we identify features of reputation domain co-occurrence

68 and the semantic content of ethnography describing reputations. The following aims guide 69 our study:

70 - Aim 1: To assess the distribution of ethnographic evidence for reputation domains.

71 - Aim 2: To identify if reputation domains exhibit systematic gender-biases and if these 72 domains vary within gender.

73 - Aim 3: To identify the structure and interrelationships of reputation domains. 
- Aim 4: To reveal the semantic content of reputation domains in the ethnographic record.

Existing work provides strong rationale for both putative universality in human reputation domains, as well as variation by social, ecological, or gender-specific pressures. Leveraging the ethnographic record in a systematic framework, despite limitations and potential biases (see Materials and Methods, Discussion), is a first step in uncovering patterns across human societies.

\section{Materials and Methods}

\section{Ethnographic sample and coding}

To accomplish our aims we relied on the electronic Human Relations Area Files (eHRAF) an online database of primary ethnographic documents. It should be noted, the ethnographic record is male-biased given the majority of ethnographers have been men and their writings and observations have generally prioritized (deliberately or not) the behaviour and social lives of men [31,32].

The eHRAF includes thousands of documents from over 300 cultures indexed by subject at the paragraph-level [33]. Users can generate a sample of ethnographic texts (i.e., paragraphs) using Boolean searches of subject codes and/or key words. Our dataset was compiled using a keyword and eHRAF's indexing system, the Outline of Cultural Materials (OCM), which associates each paragraph with any of over 700 subject codes covering a range of topics relevant for the human sciences. We conducted an "Advanced Search" of the keyword "reputation" with any of the OCM subjects: Social Personality, Personality Traits, or Status, Role, and Prestige. This search aimed to strike a balance between retrieving a generalizable yet manageable sample of the ethnography of reputations. A limitation is that our search may have omitted particular domains of reputations. See the SI for additional details.

We read the resulting 1,383 paragraphs for content, excluding those referencing reputations for groups, non-human entities, or ethnographers. We applied these inclusion criteria because our goal is to understand individual reputations within a particular culture. We then aggregated paragraphs from the same document. This resulted in a dataset containing 319 documents from 153 diverse cultures with broad geographic coverage (see Figure S1 and Table S1). These documents had a mean word count of 140 (SD of 160 and range of 14 to 1957). We refer to this as our Document dataset, which is publicly available in the Reputation Diversity Database R package [34], including bibliographic information, culture sample, and all data.

We derived, a priori, 20 reputation domains from the scientific literature on human sociality. These include: Aggressiveness, Bravery, Coercive ability, Cooperation, Cultural conformity, Honesty, Industriousness, Material capital, Medicine, Neural capital, Oration, Parental care, Prosociality, Sexual fidelity, Social capital, Social status, Sociosexuality, Somatic capital, Supernatural ability, and Teaching (see the SI for discussion on operationalization and inclusion). Each domain is operationalized as having both a positive 
114 and negative valence. For example, evidence for the reputation domain Neural capital 115 which includes reputations for generalized or specialized intelligence, special knowledge, or cognitive abilities - could be based on evidence that a given society values expertise, as well as evidence indicating that a group actively detests mental ineptitude (or vice versa). Using these operationalized reputation domains, we coded the 319 documents in the

119 Document dataset for supporting evidence across the 20 reputation domains. Authors decomposed into groups of two were allocated a subset of documents (approximately 106 per pair) to read and code, indicating supporting evidence for each domain and whether the evidence was gendered: male-specific, female-specific, or gender neutral. We did not compute inter-coder reliability measures given coders varied in experience reading and coding ethnographic texts and common inter-rater reliability statistics can produce misleadingly low reliability metrics despite relatively high levels of simple agreement for sparse matrices, such as our data [35,36]. Author-pairs compared coded data to resolve disagreements. For divergent codings the text and operational definitions were reviewed and consensus reached on the appropriate coding. The aggregated resolved codings constitute our data. See the SI for example text and coding.

\section{Data analysis}

131 The current study is primarily exploratory. We rely on descriptive and exploratory statistical approaches to accomplish our aims. We assess the cross-cultural support for each reputation domain by estimating the proportion of documents providing supporting evidence, including across our gender coding (represented as a percentage estimate). Because our Document dataset is a sample of the ethnographic record and because multiple documents often described the same culture, we incorporated uncertainty accounting for this non-independence and hierarchical structure with generalized linear mixed effects regression models (GLMM) with random effects for culture using the lme4 package [37]. Some analyses are agnostic to gender-specific codings and any coding (female-specific, male-specific, or gender neutral) counts as supporting evidence, while others account for gender-specific codings.

To estimate the frequency of supporting evidence for each reputation domain, we fit intercept-only GLMMs with random intercepts for culture, with each binary-coded reputation domain as outcomes, for all coded data (i.e., evidence for each reputation domain independent of gender-specific codings). We also fit identical models for femalespecific and male-specific evidence. These GLMMs estimate the proportion of documents providing evidence for the reputation domains (i.e., the fixed effect with $95 \% \mathrm{CI}$ ) adjusting for the non-independence of documents from the same culture (Aims 1 and 2). We also compute the percentage of cultures with at least one document providing supporting evidence for each reputation domain (independent of gender coding), with 95\% CI estimated using a cluster bootstrap and 1,000 samples with replacement (Aim 1).

Using all data, where each row represents the gender-specific evidence for each document, we assess gender-biased evidence for each reputation domain by comparing (via information criterion model selection) an intercept-only GLMM (with random intercepts for documents nested within culture and for culture language family) to similar models which include a gender-term covariate (Aim 2). 
157 We rely on hierarchical cluster analysis to identify structure (i.e., features) among

158 reputation domains (Aim 3). We then use text-analytic methods and a document-term matrix of our corpus of ethnography with penalized regression to identify semantic content

160 predictive of evidence for reputation features (Aim 4).

161 We also investigated sources of bias in our coded data due to features of the ethnographic

162 record. We used the presence of a female coauthor, document publication year, and total

163 pages of ethnography per culture in the eHRAF as predictors of our reputation domains

164 (accounting for the hierarchical document-culture structure and culture language family).

165 All analyses were conducted with R version 4.0.2 (2020-06-22).

\section{Results}

167 Evidence for reputation domains varied across subsistence types with horticulturalists and 168 agriculturalists overrepresented relative to pastoralists and hunter-gatherers (Table S1). Evidence was also male-biased. Of the 1252 counts of supporting evidence across domains, 695 (56\%) were coded as male-specific, 418 (33\%) were coded as gender neutral, and 139

171 (11\%) were coded as female-specific (Table S2).

172 Bias assessment analyses did not identify strong evidence of bias due to our meta-

173 ethnographic measures. Consequently, we did not incorporate such measures in analyses.

174 See the SI for results.

\section{Evidence for reputation domains}

176 For all 20 reputation domains, we report the percentage of cultures that provided at least one count of supporting evidence, independent of gender coding. At the culture-level, the most strongly supported domains, documented in over $50 \%$ of cultures included Cultural conformity, Neural capital, Prosociality, and Social status (Figure 1A).

180 We report the proportion of documents that provided supporting evidence including for 181 gender-specific evidence (Figure 1B). At the document-level, the most strongly supported 182 domains, represented in over $30 \%$ of documents, included Cultural conformity, Prosociality, 183 Social status, Neural capital, and Industriousness. Evidence for these reputation domains 184 was strongly male-biased, in particular Social status and Neural capital which were the 185 most supported male-specific domains. The most strongly supported female-specific 186 reputation domains (although male-biased overall) were Cultural conformity and Industriousness. The between-domain variation among female-specific evidence was minimal compared to the male-specific evidence which was more variable. We emphasize the relatively low levels of female-specific evidence could be a feature of systemic male-

191 diversity (see Discussion). 


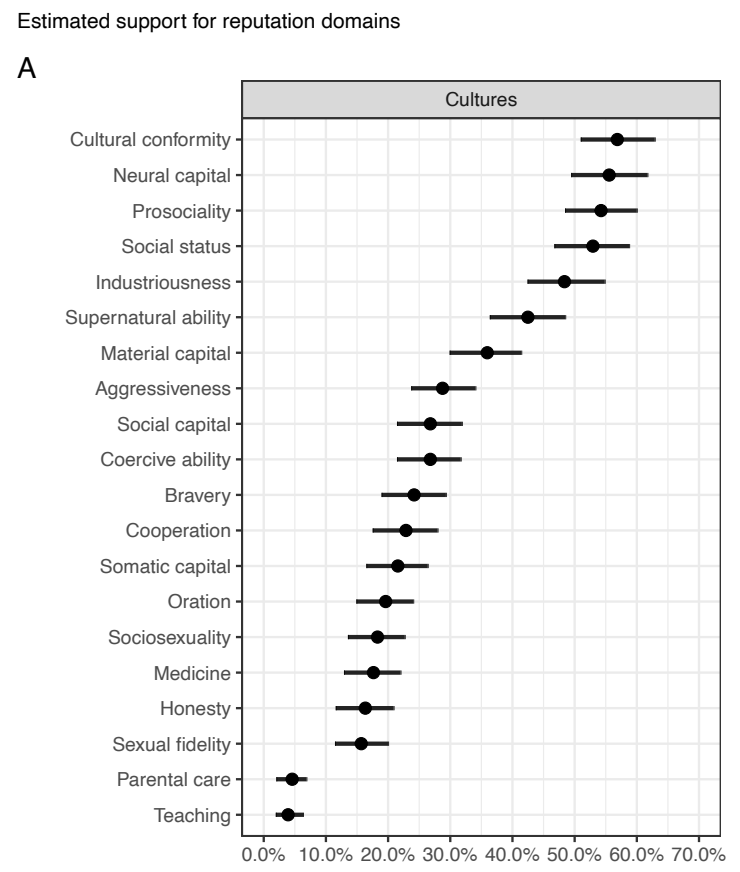

B

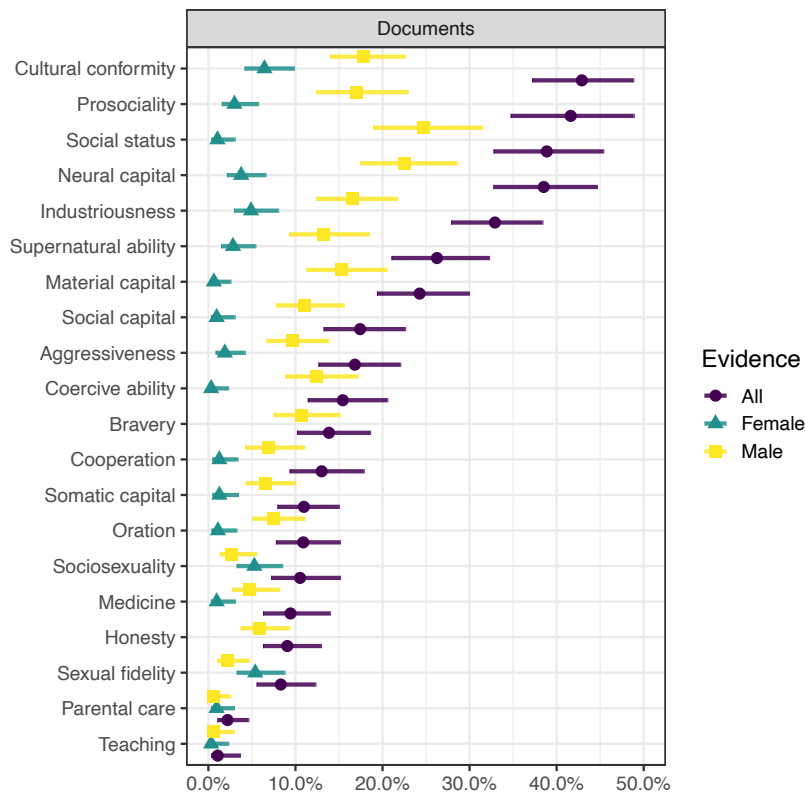

192

Figure 1: Evidence for reputation domains. A: Percentage of cultures providing at least one supporting document (95\% CI estimated using a cluster bootstrap). B: Percent of documents providing supporting evidence (95\% CI computed with intercept-only mixed effects models).

Purple circles: Estimates from all data independent of gender coding. Green triangles:

Estimates from female-specific evidence. Yellow squares: Estimates from male-specific evidence.

To assess gender-biases in the supporting evidence for reputation domains, we fit two binomial GLMMs of each reputation domain using the entire data set, where each row represents the gender-specific evidence for each document (i.e., female-specific, malespecific, gender neutral; three rows per document). The first model was an intercept-only GLMM with the binary coded reputation domains as outcomes and random intercepts for document nested within culture (to account for the repeated measures of evidence type per document and multiple documents per culture) and a random intercept for culture language family (to partially account for shared ancestry). These intercept-only models were compared to similar models which included gender-evidence type as a covariate. We compared the intercept-only models to their respective gender-term models using Akaike Information Criterion (AIC) [38]. Gender was deemed to be a predictor of reputation domain evidence when AIC $\Delta<-2$ [39]. Results are reported in Table S3 and support patterns in Figure 1B. Evidence for all domains was male-biased with the following exceptions: Sociosexuality, Parental care, and Teaching did not demonstrate gender biases and Sexual fidelity was female-biased. Two reputation domains (Bravery and Honesty) did not produce female-specific evidence and were not included. 


\section{Structural features of reputation domains}

216 Evidence for different reputation domains may co-occur within documents, putatively 217 suggesting domain interrelatedness and structure. To identify features (i.e., clusters) of 218 domains we used agglomerative hierarchical cluster analysis. See the SI for details.

219 Figure 2 displays a dendrogram from cluster analysis of the 20 reputation domains, which includes two estimates of significance for how strongly each cluster is supported by the

221 data. We rely on the AU (approximately unbiased) $p$ values (represented in red at each 222 cluster's "edge"), which are computed by multiscale bootstrap resampling and represented as percentages (clusters with AU values $>95$ are strongly supported; top-level clusters are automatically outlined by red rectangles). This revealed five strongly supported clusters we post hoc identify as Sexuality, Dominance, Supernatural healing, Social and material success, and Cultural group unity. We used these clusters to compute new variables, henceforth reputation domain features. Although the cluster capturing Neural capital and Oration was only moderately supported $(\mathrm{AU}=76)$, given Neural capital was among the most frequent domains and oratory abilities are a type of neural capital we computed a Neural capital feature from this cluster. For each document, these six reputation domain 


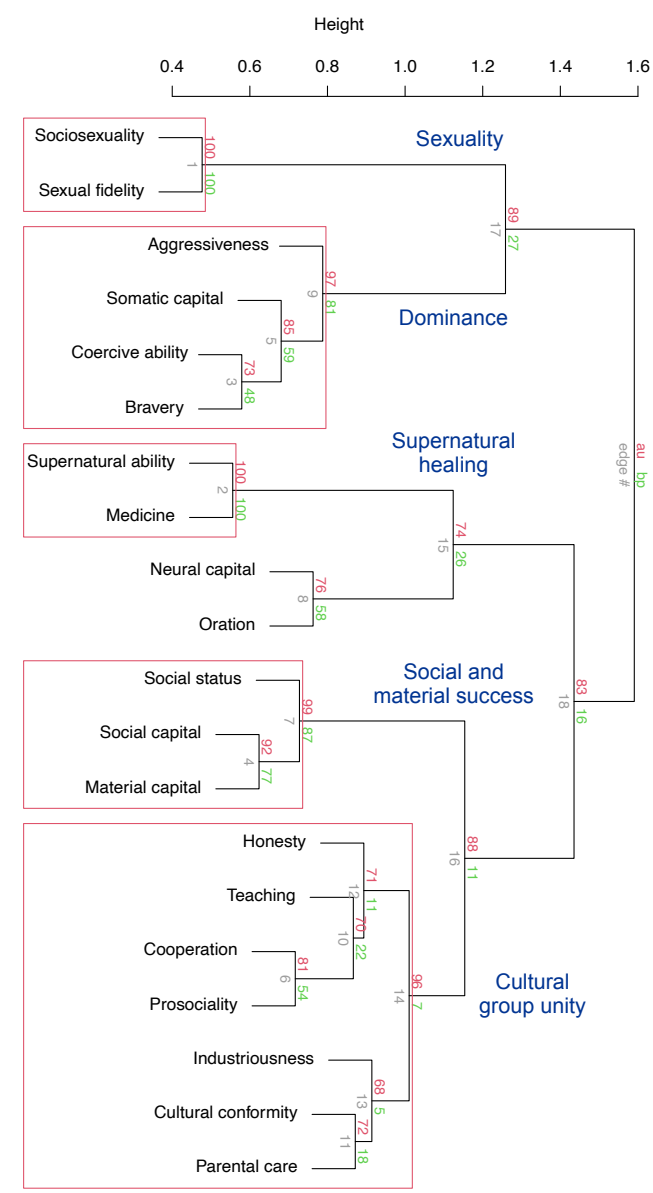

234 Figure 2: Cluster analysis of reputation domains. Distances were 1 - cor. Ward agglomeration method. AU p-values (red) computed with 10,000 bootstrap samples using the pvclust package [40]. Edge number in grey.

237 We estimated the percentage of cultures providing support for each reputation domain feature using the same cluster bootstrap methods used to estimate the culture-level support for domains. Supporting evidence for the Cultural group unity and Social and material success features was common across cultures, documented in $82 \%$ and $64 \%$ of cultures, respectively. Evidence for the Neural capital feature was documented in $59 \%$ of cultures, the Dominance feature in 53\% of cultures, the Supernatural healing feature in $24344 \%$ of cultures, and the Sexuality feature in 23\% of cultures (Figure 3). 


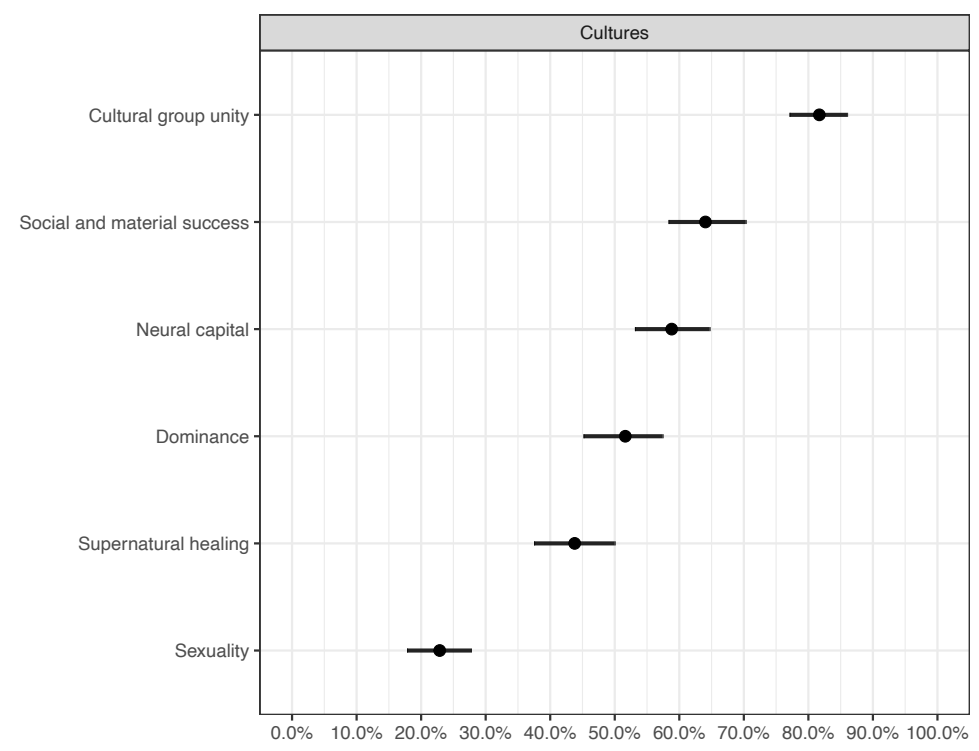

245 Figure 3: Percentage of cultures providing evidence for reputation features (95\% CI estimated 246 via cluster bootstrap).

\section{The ethnography of reputation domains}

248 We used text analysis to explore the ethnography of reputation domains in reference to our six features. We created a document-term matrix (DTM) of all "informative" words in our corpus of texts which captures the frequency of each unique term within each document. We fit an elastic net logistic regression model (with the lasso penalty, $\alpha=1$ ) of each of the six features as a function of the frequencies of all 8,770 unique words (using the glmnet package [41]). Words that were strong positive predictors epitomized the semantic content of documents which provided evidence for that feature. Figure 4 displays non-zero coefficients from elastic net lasso regression models of each reputation domain feature.

Evidence for the Cultural group unity feature was positively predicted by terms related to social relationships and community (e.g., family, person, wife) and negatively predicted by terms related to the supernatural (e.g., spirit, shaman). Evidence for the Social and material success feature was positively predicted by wealth, prestige, and terms for leadership and status. Evidence for the Neural capital feature was positively predicted by skill, leader, and village. Evidence for the Dominance feature was positively predicted by war, strong, kill, and physical implicating reputations for dominance with conflict, physical formidability, and aggression. Negative predictors of the Dominance feature included status and wealth, suggesting a distinction between dominance and prestige. Evidence for the Supernatural healing feature was positively predicted by the terms, shaman, cure, power, and medicine; woman was a weak negative predictor. Evidence for the Sexuality feature was predicted by girl, woman, and sexual. 
Text analytic results

A

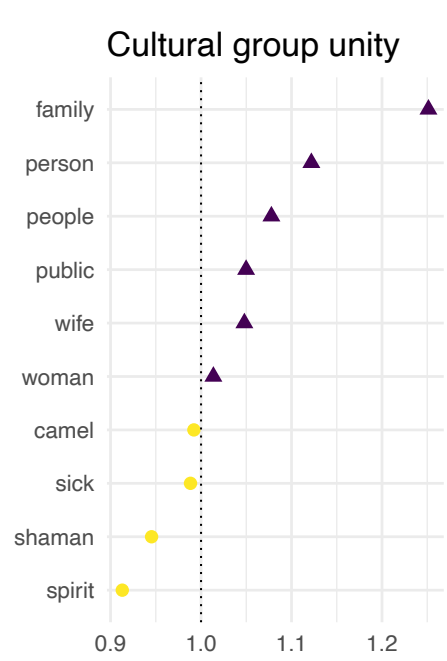

D

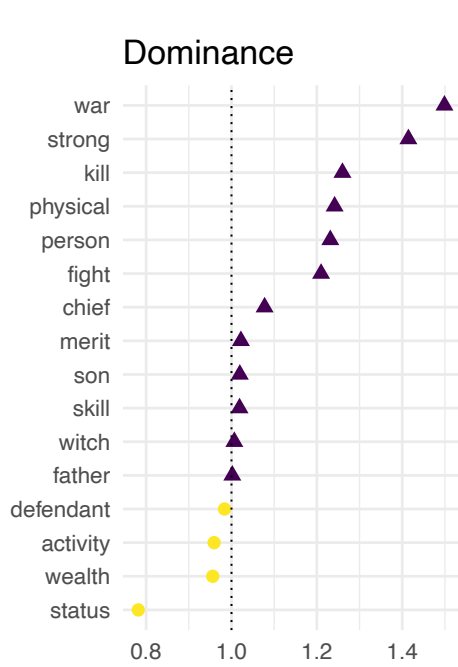

B

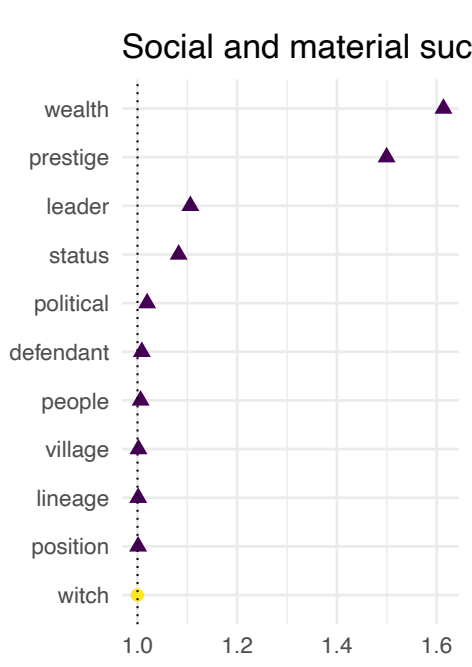

$\mathrm{E}$

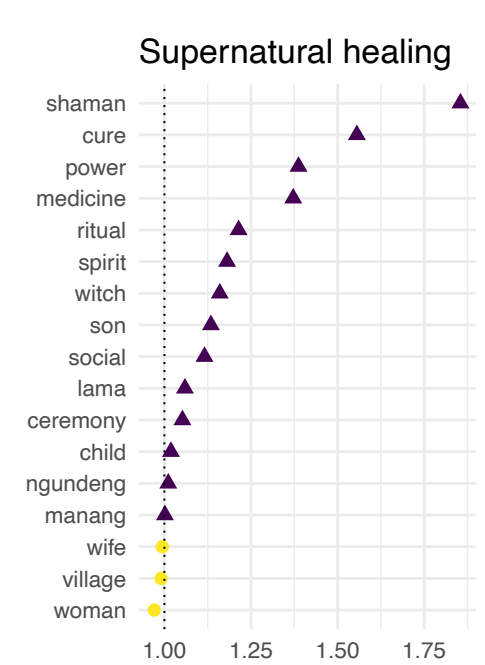

C

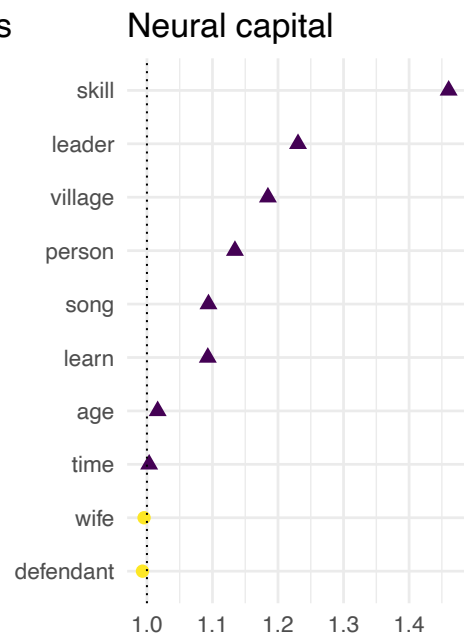

$\mathrm{F}$

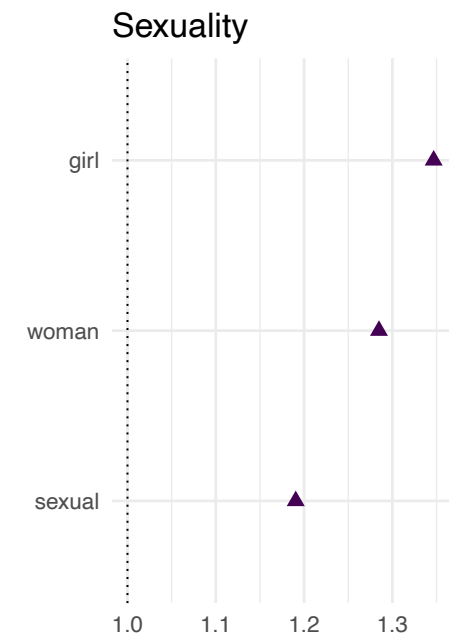

Figure 4: Non-zero coefficients from text analysis elastic net regression models of evidence for reputation features. Coefficients indicate the words in each document which best predicted evidence for the feature. Positive coefficients as purple triangles. Negative coefficients as yellow circles.

\section{Discussion}

274 The content, structure, and diversity of reputation domains across societies are understudied from a holistic perspective. The current study was motivated by a lack of cross-cultural research, despite widespread theorizing in biology, psychology and anthropology regarding the role of reputations for sociality and evolutionary dynamics. Using the eHRAF database we extracted ethnographic accounts of individual-level reputation domains. Results suggest: 1 ) there is considerable cross-cultural variability in evidence for reputation domains - some domains are common in the ethnographic record (e.g., cultural conformity, prosociality) while others are relatively rare (e.g., teaching, 
honesty); 2) evidence for most reputations are male-biased with male-specific reputation domains more variable than female-specific domains; and 3) reputation domains cluster within six features: cultural group unity, dominance, neural capital, sexuality, social and material success, and supernatural healing. Below we interpret results from an evolutionary social science perspective.

\section{Diversity in reputation domains}

288 Most reputation domains (16 of 20) were documented in less than half of sampled cultures (Figures 1A and 3). Despite variability, some were more common than others, including reputations for cultural conformity, prosociality, social status, neural capital, and industriousness. These results are notable because of what is missing: "cooperation." Evolutionary-oriented scholars have implicated cooperative reputations for explaining human ultrasociality $[5,13,14]$, yet reputations for "cooperativeness" were documented in only $23 \%$ of cultures. Reputations indirectly related to cooperation (e.g., conformity, honesty, social relationships, and industriousness), however, were common across societies. Reputations for cooperation were also captured by the most common feature: cultural group unity (Figure 3). The limited evidence of reputations for cooperativeness could be due in part to the nature of the ethnographic record (see Limitations) or a product of our operational definition. We follow developmental and neuro-psychologists $[23,42,43]$ by differentiating cooperation - defined as the likelihood an individual intentionally assists another in order to achieve a joint goal - from prosociality - defined as the likelihood one will invest in group welfare or act in group-altruistic ways (see the SI for discussion).

While we wish to avoid sweeping claims and emphasize the exploratory nature of our study, these results signal a need to expand research on reputations beyond cooperativeness, incorporating a variety of domains and examining their effect on sociality, particularly in experimental settings (sensu, [3]). Across cultures, distinct reputations capturing inter-individual variation in personality, experiences, capacities, and reliability, likely underpin much of human sociality, including cooperativeness.

\section{Gender-differences in reputation domains}

Evidence for most reputation domains was male-biased and there was greater variance among male than female reputation domains (Figure 1B). While this finding is consistent with research demonstrating that, across cultures, male social life is typically more public than female social life [44-46] we cannot disentangle male-bias in ethnography from putative male biases in more overt sociality and reputation diversity. This male-biased pattern is consistent, however, with perspectives suggesting societies disproportionately channel opportunities to men to differentiate themselves, at the detriment of women who have fewer avenues to develop social capital [30,45,47,48]. As Rosaldo ([46], pp. 393-394) suggests reviewing much ethnography, "the vast majority of opportunities for public influence and prestige, the ability to forge relationships, determine enmities, speak up in public, use or forswear the use of force are all recognized as men's privilege and right."

321 Competition among women, however, has been suggested to be more indirect and 322 reputation-based, compared to men [25,49], which would predict at least some female- 
specific reputation domains or limited variance between reputation domains of women and men. Some empirical studies of gender-differences in social influence among relatively egalitarian societies have found similarity in the weights of particular status-determining attributes between genders, despite male biases in overall influence [48,50]. Future comparative studies should more comprehensively define female-specific reputation domains and design targeted methods to document supporting evidence [51,52].

The only reputation domain more strongly associated with women than men was sexual fidelity; reputations for sociosexuality did not demonstrate gender-bias (see [53] for similar results). These findings support evolutionary psychology models drawing on sexual selection theory which predict gender-specific evaluations related to reproductive strategies $[27,49]$ and widespread male reproductive skew specific to influential men [36,54-56]. Overall, reputations related to sexuality were rare in our data. Sexuality may have been a taboo topic in some ethnographic contexts, but the ethnographic record includes rich descriptions of human sexuality $[57,58]$. It is possible that our search strategy did not capture much of the ethnography of reputations related to sexuality. Nonetheless, findings do not provide support for a universal psychology dedicated to evaluating female sexuality vis-à-vis males and do support perspectives emphasizing flexibility in reputations, strategies, and norms related to sexuality [59].

\section{Reputation domain structure and evolutionary theories}

We find reputation domains are structured along six features which we termed Cultural group unity, Social and material success, Neural capital, Dominance, Supernatural healing, and Sexuality. This data-driven, exploratory analysis comports well with theory from evolutionary psychology and the framework of human uniqueness in evolutionary anthropology.

Evolutionary psychologists examining the content of competitor derogation [27], have predicted men will often be evaluated for abilities to control resources necessary for status achievement, attracting mates, and reproductive success. We find some support for this claim given the reputation features of Dominance and Social and material success. Additionally, evidence for the reputation domains Social status, Material capital, and Coercive ability were among the most male-biased domains (Figure 1B). Status hierarchies shape priority of access to resources and scholars have suggested they can be navigated through two distinct (though non-mutually exclusive) pathways: dominance and prestige $[7,60,61]$. These results support a distinction between dominance and social status or prestige [62,63], indicated by the cluster and text analyses (Figures 3, 4C and 4D).

Reputations for prestige (our Social and material success feature) are associated with social networks as well as material resources, more so than reputations for dominance (Figures 2, 4B). These results are consistent with analyses among the Tsimane illustrating interrelationships between status, social networks, and social and material gains from cooperation with high status individuals [64]. Results also support associations between reputations for dominance and coercion, physical aggression, and conflict (Figure 4D) [65]. Reputations for bravery were also captured by the Dominance feature and cross-cultural research identified bravery as a universal feature of prosocial moral values [66]. Taken 
together, these results suggest reputations for social status and prestige are often associated with capacities for resource control while reputations for dominance may, in some contexts, be associated with prosocial investments [63,67-69].

368 The clustering of reputations for cooperation, prosociality, conformity, honesty, teaching, and industriousness fits conceptions of the distinct nature of human social cognition, as well as fundamental structures of human groups. For example, scholars suggest human uniqueness relies on an evolved psychology dedicated to reasoning about others having cooperative and prosocial motivations $[42,43,70]$. These models suggest cultural conformity and learning biases leads to the evolution of well-structured groups and better equip groups to compete with others groups $[10,71,72]$. Such between-group competitive dynamics can occur through altruistic provisioning of group members or through intergroup violence $[73,74]$ and can in turn, further support within group cooperation $[75,76]$.

378 Lastly, the supernatural healing feature is associated with unique features of the human niche (i.e., religion) and fits long-standing anthropological notions about the important role benefits for and impose costs on group members $[68,77-79]$.

\section{Limitations}

Our study has several limitations. First, our data are limited to the content ethnographers recorded and published. Information on reputations that the ethnographer was unaware of, not interested in, nor permitted to research, constrains available data. Therefore, while we can conclude the widespread ethnographic evidence of some reputation domains likely indicates their cross-cultural importance, we cannot conclude reputations domains lacking substantial evidence are indeed rare across cultures. Additionally, the terms an ethnographer uses for reputation domains may reflect their worldview (etic), rather than the worldview of the focus population (emic). We attempted to assess potential biases in our data due to meta-ethnographic measures (see the SI), however, it is possible other features of ethnography or ethnographers influenced results.

Ethnographic materials related to the social, economic, and cultural lives of women are systematically underreported, especially in the early history of the field $[30,31,80]$. Thus, the extent to which women have fewer avenues for gaining reputations cross-culturally remains unclear and cannot be evaluated via these methods. However, the evidence of gender biases we discovered comport with the common notion that patriarchy is pervasive globally and negatively impacts women's ability to achieve recognition, political power, economic capital, and autonomy (see [81]).

We identified the 20 reputation domains a priori, drawing on the literature on human uniqueness and sexual selection theory, which itself is likely to be biased by authors and general biases across the human sciences. While a useful starting point for exploring reputational diversity, we imagine that other domains could exist. Lastly, we constrained our eHRAF search using the keyword "reputation", which could have missed other content on reputations that used adjacent language (e.g., personality, gossip). Recognizing these 
limitations, these results provide greater cross-cultural validity to existing theories of reputation and can spark future empirical and theoretical work better incorporating the cultural diversity, structure, and gendered dimensions of reputation domains.

\section{Conclusion}

410 Reputations are a critical component of human social life and have fundamental 411 implications for human evolution. From a socio-structural perspective, reputations are the 412 pathways by which societies evaluate individuals and are the mechanisms through which individuals can distinguish themselves. Despite their centrality to much of human sociality, little systematic cross-cultural research exists on the content and structure of reputation domains. We find that ethnographic evidence for reputations is variable across societies, tends to focus on cultural conformity and prosociality, displays large gender biases with greater variance among males, and is structured around themes related to human uniqueness.

Drawing on Chapais' [82] distinction between context-independent vs. context-dependent human universals, we hypothesize reputations for cultural group unity will be a contextindependent universal, likely to manifest in all human societies; whereas reputations for social and material success, neural capital, and dominance are more likely to be contextdependent universals, promoted or suppressed by socio-ecological or cultural evolutionary processes.

\section{5}

\section{Acknowledgements}

We thank Nicole Hess and Chris von Rueden for helpful comments on this manuscript as well as the editors and two anonymous reviewers for their careful review and useful feedback.

Original data produced for the current study are available in the archived reputationdiversitydata $\mathrm{R}$ package (DOI: 10.5281/zenodo.4740791).

432 This work was supported in part by the Global Change and Sustainability Center and the Office of Undergraduate Research at the University of Utah. Zachary H. Garfield acknowledges IAST funding from the French National Research Agency (ANR) under the Investments for the Future (Investissements d'Avenir) program, grant ANR-17-EURE-0010.

438 2. Barclay P. 2015 Reputation. In The Handbook of Evolutionary Psychology (ed D Buss), pp.

\section{References}

440 3. Macfarlan SJ, Lyle HF. 2015 Multiple reputation domains and cooperative behaviour in 
442 4. Barclay P. 2013 Strategies for cooperation in biological markets, especially for humans. 443 Evolution and Human Behavior 34, 164-175. (doi:10.1016/j.evolhumbehav.2013.02.002)

444 5. Milinski M. 2016 Reputation, a universal currency for human social interactions.

445 Philosophical Transactions of the Royal Society B: Biological Sciences 371, 20150100.

446 (doi:10.1098/rstb.2015.0100)

447 6. Sugden R. 2005 The Economics of Rights, Co-operation and Welfare. Palgrave Macmillan 448 UK. (doi:10.1057/9780230536791)

449 7. Henrich J, Gil-White FJ. 2001 The evolution of prestige: Freely conferred deference as a mechanism for enhancing the benefits of cultural transmission. Evolution and Human 451 Behavior 22, 165-196.

452 8. Buss DM, Durkee PK, Shackelford TK, Bowdle BF, Schmitt DP, Brase GL, Choe JC, 453 Trofimova I. 2020 Human status criteria: Sex differences and similarities across 14 nations. 454 Journal of Personality and Social Psychology, No Pagination Specified-No Pagination 455 Specified. (doi:10.1037/pspa0000206)

9. Glowacki L. 2020 The Emergence of locally adaptive institutions: Insights from traditional social structures of East African pastoralists. Biosystems, 104257. (doi:10.1016/j.biosystems.2020.104257)

10. Chudek M, Henrich J. 2011 Culture-gene coevolution, norm-psychology and the emergence of human prosociality. Trends Cogn Sci 15, 218-26.

461 (doi:10.1016/j.tics.2011.03.003)

462 11. Gintis H. 2000 Strong Reciprocity and Human Sociality. Journal of Theoretical Biology 463 206, 169-179. (doi:10.1006/jtbi.2000.2111)

464 12. Subiaul F, Vonk J, Okamoto-Barth S, Barth J. 2008 Do chimpanzees learn reputation by 465 observation? Evidence from direct and indirect experience with generous and selfish strangers. Animal Cognition 11, 611-623. (doi:10.1007/s10071-008-0151-6) 393, 573-577. (doi:10.1038/31225) Exchange Variation in a Dominican Community. Current Anthropology 53, 118-124.

472 15. Hess NH, Hagen EH. 2006 Psychological adaptations for assessing gossip veracity.

473 Human Nature 17, 337-354.

474 16. Giardini F, Wittek R. 2019 The Oxford handbook of gossip and reputation. New York: 475 Oxford University Press. 
17. Enquist M, Ghirlanda S, Eriksson K. 2011 Modelling the evolution and diversity of cumulative culture. Philosophical Transactions of the Royal Society B: Biological Sciences 366, 412-423. (doi:10.1098/rstb.2010.0132)

18. Barclay P. 2004 Trustworthiness and competitive altruism can also solve the 'tragedy of the commons'. Evolution and Human Behavior 25, 209-220.

(doi:10.1016/j.evolhumbehav.2004.04.002)

19. Panchanathan K, Boyd R. 2004 Indirect reciprocity can stabilize cooperation without the second-order free rider problem. Nature 432, 499.

20. Macfarlan SJ, Quinlan R, Remiker M. 2013 Cooperative behaviour and prosocial reputation dynamics in a Dominican village. Proceedings of the Royal Society B: Biological Sciences 280, 20130557. (doi:10.1098/rspb.2013.0557)

21. Lyle HF, Smith EA. 2014 The reputational and social network benefits of prosociality in an Andean community. Proceedings of the National Academy of Sciences 111, 4820-4825. (doi:10.1073/pnas.1318372111)

22. Hawkes K, Bird RB. 2002 Showing off, handicap signaling, and the evolution of men's work. Evolutionary Anthropology 11, 58-67.

23. Fiske ST, Cuddy AJC, Glick P. 2007 Universal dimensions of social cognition: Warmth and competence. Trends in Cognitive Sciences 11, 77-83. (doi:10.1016/j.tics.2006.11.005)

24. Benard S. 2013 Reputation systems, aggression, and deterrence in social interaction. Social Science Research 42, 230-245. (doi:10.1016/j.ssresearch.2012.09.004)

25. Hess NH, Hagen EH. 2006 Sex differences in indirect aggression Psychological evidence from young adults. Evolution and Human Behavior 27, 231-245.

26. Hess NH, Hagen EH. 2019 Gossip, reputation, and friendship in within-group competition: An evolutionary perspective. In Handbook of Reputation and Gossip, Oxford University Press.

27. Buss DM, Dedden LA. 1990 Derogation of Competitors. Journal of Social and Personal Relationships 7, 395-422. (doi:10.1177/0265407590073006)

28. Davis A, Vaillancourt T, Arnocky S, Doyel R. 2019 Women's Gossip as an Intrasexual Competition Strategy. In The Oxford Handbook of Gossip and Reputation (eds F Giardini, R Wittek), (doi:10.1093/oxfordhb/9780190494087.013.16)

29. Nowak MA. 2006 Five rules for the evolution of cooperation. Science (New York, N.y.) 314, 1560-1563. (doi:10.1126/science.1133755)

30. Post ER, Macfarlan SJ. 2020 Tracking Cross-Cultural Gender Bias in Reputations. CrossCultural Research 54, 346-363. (doi:10.1177/1069397120910429) 
510 31. Mukhopadhyay CC, Higgins PJ. 1988 Anthropological studies of women's status 511 revisited: 1977-1987. Annu Rev Anthropol 17, 461-95.

512 (doi:10.1146/annurev.an.17.100188.002333)

513 32. Abu-Lughod L. 1990 Can There Be A Feminist Ethnography? Women \& Performance: a

514 journal of feminist theory 5, 7-27. (doi:10.1080/07407709008571138)

515 33. Ember CR. 2007 Using the HRAF Collection of Ethnography in Conjunction With the 516 Standard Cross-Cultural Sample and the Ethnographic Atlas. Cross-Cultural Research 41, 517 396. (doi:10.1177/1069397107306593)

518 34. Garfield Z, Macfarlan S, Schacht R, Post E, Ingram D, Uehling A. 2021

519 Zhgarfield/reputationdiversitydata: Initial release of the reputationdiversitydata package.

520 (doi:10.5281/zenodo.4740791)

521 35. Syme KL, Garfield ZH, Hagen EH. 2015 Testing the bargaining vs. Inclusive fitness 522 models of suicidal behavior against the ethnographic record. Evolution and Human 523 Behavior 37, 179-192. (doi:10.1016/j.evolhumbehav.2015.10.005)

524 36. Garfield ZH, Hubbard H Robert, Hagen EH. 2019 Evolutionary models of leadership:

525 Tests and synthesis. Human Nature 30, 23-58. (doi:10.1007/s12110-019-09338-4)

526 37. Bates D, Mächler M, Bolker B, Walker S. 2015 Fitting linear mixed-effects models using 527 Ime4. Journal of Statistical Software 67, 1-48. (doi:10.18637/jss.v067.i01)

528 38. Burnham KP, Anderson DR, Huyvaert KP. 2011 AIC model selection and multimodel inference in behavioral ecology: Some background, observations, and comparisons.

530 Behavioral Ecology and Sociobiology 65, 23-35. (doi:10.1007/s00265-010-1029-6)

531 39. Burnham KP, Anderson DR, editors. 2002 Model selection and multimodel inference: A

532 practical information-theoretic approach. Springer New York. (doi:10.1007/b97636)

533 40. Suzuki R, Shimodaira H. 2015 Pvclust: Hierarchical Clustering with P-Values via

534 Multiscale Bootstrap Resampling. See https://CRAN.R-project.org/package=pvclust.

535 41. Zou H, Hastie T. 2005 Regularization and variable selection via the elastic net. Journal of 536 the Royal Statistical Society: Series B (Statistical Methodology) 67, 301-320.

537 (doi:10.1111/j.1467-9868.2005.00503.x)

538 42. Tomasello M, Carpenter M, Call J, Behne T, Moll H. 2005 Understanding and sharing 539 intentions: The origins of cultural cognition. Behavioral and Brain Sciences 28, 675-691.

540 (doi:10.1017/S0140525X05000129)

541 43. MacLean EL. 2016 Unraveling the evolution of uniquely human cognition. Proceedings

542 of the National Academy of Sciences 113, 6348-6354. (doi:10.1073/pnas.1521270113)

543 44. Hawkes K et al. 1993 Why Hunter-Gatherers Work: An Ancient Version of the Problem 544 of Public Goods [and Comments and Reply]. Current Anthropology, 341-361. 
545 45. Smith JE, Ortiz CA, Buhbe MT, van Vugt M. 2020 Obstacles and opportunities for female 546 leadership in mammalian societies: A comparative perspective. Special issue on Evolution

547 and Biology of Leadership 31, 101267. (doi:10.1016/j.leaqua.2018.09.005)

548 46. Rosaldo MZ. 1980 The use and abuse of anthropology: Reflections on feminism and 549 cross-cultural understanding. Signs 5, 389-417.

550 47. Lin N. 2000 Inequality in Social Capital. Contemporary Sociology 29, 785-795.

$551 \quad$ (doi:10.2307/2654086)

552 48. von Rueden C, Alami S, Kaplan H, Gurven M. 2018 Sex differences in political leadership 553 in an egalitarian society. Evolution and Human Behavior

554 (doi:10.1016/j.evolhumbehav.2018.03.005)

555 49. Campbell A. 2002 A mind of her own: The evolutionary psychology of women. Oxford 556 University Press Oxford.

557 50. Garfield ZH, Hagen EH. 2020 Investigating evolutionary models of leadership among 558 recently settled Ethiopian hunter-gatherers. Special issue on Evolution and Biology of 559 Leadership 31, 101290. (doi:10.1016/j.leaqua.2019.03.005)

560 51. Dahlberg F. 1981 Woman the gatherer. New Haven: Yale University Press.

561 52. Low BS. 2005 Women's lives there, here, then, now: A review of women's ecological and 562 demographic constraints cross-culturally. Evolution and Human Behavior 26, 64-87.

563 (doi:10.1016/j.evolhumbehav.2004.08.011)

564 53. Schacht R, Borgerhoff Mulder M. 2015 Sex ratio effects on reproductive strategies in 565 humans. Royal Society Open Science 2, 140402. (doi:10.1098/rsos.140402)

566 54. von Rueden C, Jaeggi AV. 2016 Mens status and reproductive success in 33

567 nonindustrial societies: Effects of subsistence, marriage system, and reproductive strategy.

568 Proceedings of the National Academy of Sciences, 201606800.

569 55. Schacht R, Bell AV. 2016 The evolution of monogamy in response to partner scarcity.

570 Scientific Reports 6, 32472. (doi:10.1038/srep32472)

571 56. Schacht R, Rauch KL, Borgerhoff Mulder M. 2014 Too many men: The violence problem?

572 Trends Ecol Evol 29, 214-22. (doi:10.1016/j.tree.2014.02.001)

573 57. Hames R, Garfield ZH, Garfield M. 2017 Is Male Androphilia a Context-Dependent Cross574 Cultural Universal? Archives of Sexual Behavior 46, 63-71. (doi:10.1007/s10508-016-0855575 7)

576 58. Malinowski B. 1932 The sexual life of savages. London: Routledge.

577 59. Hrdy SB. 2000 The optimal number of fathers. Evolution, demography, and history in 578 the shaping of female mate preferences. Ann N Y Acad Sci 907, 75-96. 
579 60. Van Vugt M, Smith JE. 2019 A Dual Model of Leadership and Hierarchy: Evolutionary 580 Synthesis. Trends in Cognitive Sciences 23, 952-967. (doi:10.1016/j.tics.2019.09.004)

581 61. Cheng JT. 2019 Dominance, Prestige, and the Role of Leveling in Human Social 582 Hierarchy and Equality. Current Opinion in Psychology (doi:10.1016/j.copsyc.2019.10.004)

583 62. Cheng JT, Tracy JL, Foulsham T, Kingstone A, Henrich J. 2013 Two ways to the top: 584 Evidence that dominance and prestige are distinct yet viable avenues to social rank and 585 influence.J Pers Soc Psychol 104, 103-25. (doi:10.1037/a0030398)

586 63. Von Rueden C. 2014 The roots and fruits of social status in small-scale human societies. In The psychology of social status (eds JT Cheng, JL Tracy, C Anderson), pp. 179-200.

588 Springer.

589 64. von Rueden CR, Redhead D, O'Gorman R, Kaplan H, Gurven M. 2019 The dynamics of 590 men's cooperation and social status in a small-scale society. Proceedings of the Royal Society 591 B: Biological Sciences 286, 20191367. (doi:10.1098/rspb.2019.1367)

592 65. Earle TK. 1997 How chiefs come to power: The political economy in prehistory. Stanford 593 University Press.

594 66. Curry OS, Mullins DA, Whitehouse H. 2019 Is It Good to Cooperate?: Testing the Theory 595 of Morality-as-Cooperation in 60 Societies. Current Anthropology 60, 47-69.

596 (doi:10.1086/701478)

597 67. Durkee PK, Lukaszewski AW, Buss DM. 2020 Psychological foundations of human status 598 allocation. Proceedings of the National Academy of Sciences 117, 21235-21241.

599 (doi:10.1073/pnas.2006148117)

600 68. Garfield ZH, Syme KL, Hagen EH. 2020 Universal and variable leadership dimensions 601 across human societies. Evolution and Human Behavior 41, 397-414.

602 (doi:10.1016/j.evolhumbehav.2020.07.012)

603 69. Pietraszewski D. 2020 The evolution of leadership: Leadership and followership as a 604 solution to the problem of creating and executing successful coordination and cooperation 605 enterprises. Special issue on Evolution and Biology of Leadership 31, 101299.

606 (doi:10.1016/j.leaqua.2019.05.006)

607 70. Hill K, Barton M, Hurtado AM. 2009 The emergence of human uniqueness: Characters 608 underlying behavioral modernity. Evolutionary Anthropology: Issues, News, and Reviews 18, 609 187-200. (doi:10.1002/evan.20224)

610 71. Boyd R_, Richerson P. 1985 Culture and the Evolutionary Process. Chicago: University of 611 Chicago Press.

612 72. Henrich J, Boyd R. 1998 The Evolution of Conformist Transmission and the Emergence 613 of Between-Group Differences. Evolution and Human Behavior 19, 215-241.

614 (doi:10.1016/S1090-5138(98)00018-X) 
615 73. Bowles S. 2006 Group Competition, Reproductive Leveling, and the Evolution of Human 616 Altruism. Science 314, 1569-1572. (doi:10.1126/science.1134829)

617 74. Glowacki L, Wilson ML, Wrangham RW. 2020 The evolutionary anthropology of war. 618 Journal of Economic Behavior \& Organization 178, 963-982.

619 (doi:10.1016/j.jebo.2017.09.014)

620 75. Gavrilets S, Fortunato L. 2014 A solution to the collective action problem in between621 group conflict with within-group inequality. Nature communications 5, 3526.

622 76. Henrich J, Chudek M, Boyd R. 2015 The Big Man Mechanism: How prestige fosters

623 cooperation and creates prosocial leaders. Phil. Trans. R. Soc. B 370, 20150013.

624 77. Singh M, Kaptchuk TJ, Henrich J. 2020 Small gods, rituals, and cooperation: The 625 Mentawai water spirit Sikameinan. Evolution and Human Behavior

626 (doi:10.1016/j.evolhumbehav.2020.07.008)

627 78. Singh M. 2017 The cultural evolution of shamanism. The Behavioral and brain sciences 628 2017, 1-83.

629 79. Lightner AD, Heckelsmiller C, Hagen EH. 2021 Ethnoscientific expertise and knowledge 630 specialisation in 55 traditional cultures. Evolutionary Human Sciences, 1-52.

631 (doi:10.1017/ehs.2021.31)

632 80. Rosaldo MZ. 1974 Woman, culture, and society: A theoretical overview. In Woman, 633 culture and society (eds MZ Rosaldo, L Lamphere), pp. 17-43. Stanford, CA: Stanford 634 University Press.

635 81. United Nations Sustainable Development. In press. Gender equality and women's 636 empowerment. See https://www.un.org/sustainabledevelopment/gender-equality/ 637 (accessed on 27 June 2021).

638 82. Chapais B. 2014 Complex Kinship Patterns as Evolutionary Constructions, and the 639 Origins of Sociocultural Universals. Current Anthropology 55, 751-783.

640 (doi:10.1086/678972) 